\title{
One-magnon and exciton inelastic light scattering in the antiferromagnet $\mathrm{CoF}_{2}$
}

\author{
E. Meloche ${ }^{*}$ and M.G. Cottam \\ Department of Physics and Astronomy, University of Western Ontario, London, Ontario N6A 3K7, Canada \\ D.J. Lockwood \\ Institute for Microstructural Sciences, National Research Council, Ottawa, Ontario K1A OR6, Canada \\ E-mail: David.Lockwood@nrc-cnrc.gc.ca
}

Received July 5, 2013

\begin{abstract}
Experimental data are reported for the temperature and polarization dependence of the one-magnon Raman light scattering in the rutile-structure antiferromagnet $\mathrm{CoF}_{2}$ (Néel temperature $T_{N}=38 \mathrm{~K}$ ). The low-lying excitons are also investigated at low temperatures and comparisons made with results from earlier Raman, infrared, and neutron scattering work. A detailed analysis of the one-magnon Stokes and anti-Stokes Raman spectra is presented resulting in comprehensive data for the temperature variation up to $T_{N}$ of the one-magnon frequency, line width, and integrated intensity. A theory of the one-magnon scattering and other magnetic transitions is constructed based mainly on a spin $S=3 / 2$ exchange model, extending a simpler effective $S=1 / 2$ approach. The excitation energies and spectral intensities over a broad range of temperatures are obtained using a Green's function equation of motion method that allows for a careful treatment of the single-ion anisotropy. Overall the $S=3 / 2$ theory compares well with the experimental data.
\end{abstract}

PACS: 75.30.Ds Spin waves;

72.10.Bb Scattering by phonons, magnons, and other nonlocalized excitations;

78.30.-j Infrared and Raman spectra;

75.50.Ee Antiferromagnetics.

Keywords: antiferromagnet, inelastic light scattering, magnetic excitations.

\section{Introduction}

In an earlier paper [1] we presented a thorough comparison between experiment and theory for two-magnon inelastic light scattering in the rutile-structure antiferromagnets $\mathrm{NiF}_{2}$ and $\mathrm{CoF}_{2}$ thus complementing earlier work on isomorphic compounds such as $\mathrm{FeF}_{2}$ and $\mathrm{MnF}_{2}$ that are broadly similar magnetically [2]. The focus of the present paper is on the one-magnon light scattering, which provides further insight into the spin dynamics of these compounds because it emphasizes the magnetic excitations near the center of the Brillouin zone. By contrast, in twomagnon scattering, the excitations of magnon pairs at large wave vectors are dominant. By comparison with $\mathrm{FeF}_{2}$ and $\mathrm{MnF}_{2}$, neither $\mathrm{NiF}_{2}$ or $\mathrm{CoF}_{2}$ were well understood with regards to the temperature and polarization dependence of their one-magnon excitations, as studied through their fre- quencies and Raman intensities. They present quite distinct cases to one another, because in $\mathrm{NiF}_{2}$ there is a spin canting from true antiferromagnetic alignment, leading to a major effect on the zone-center magnons and thereby on the one-magnon light scattering, as we reported recently [3]. On the other hand, the one-magnon light scattering in $\mathrm{CoF}_{2}$ has other distinctive properties. There is no canting, but the effects arising due to a strong orbital angular momentum and a large single-ion anisotropy are dominant. This has motivated our experimental and theoretical studies presented here.

The crystallographic unit cell of $\mathrm{CoF}_{2}$ is depicted in Fig. 1 together with the relevant exchange parameters employed in this work. The antiferromagnetic ordering of the spins in $\mathrm{CoF}_{2}$ was first determined by Erickson [4] using neutron diffraction and also confirmed through studies of

* Current address: 7801 Computer Ave., Bloomington, MN 55435 


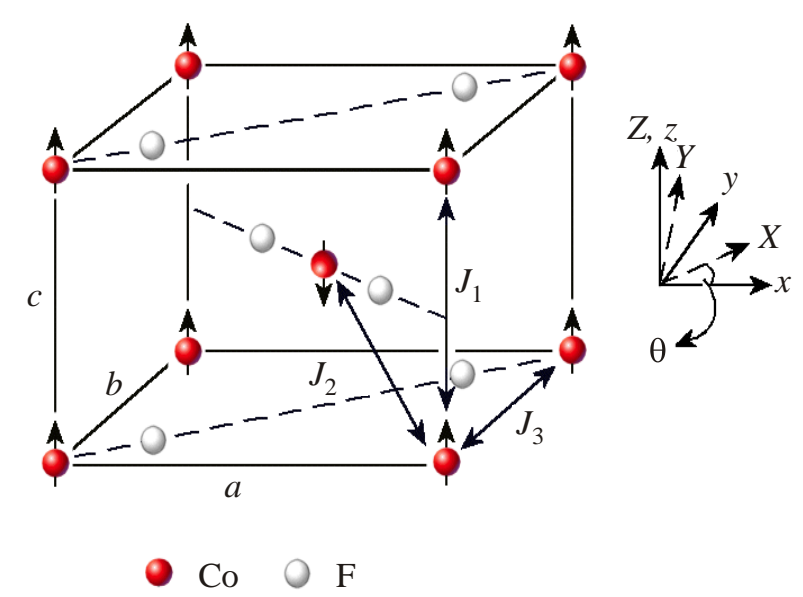

Fig. 1. The crystallographic unit cell of $\mathrm{CoF}_{2}(a=b \neq c)$ with the principal exchange interactions $J_{1}, J_{2}$ and $J_{3}$. Crystal axes $(x, y, z)$ and laboratory axes $(X, Y, Z)$ are illustrated in relation to the unit cell. The $X$ and $Y$ directions are orthogonal to the $c(Z)$ axis but are rotated by $\approx 45^{\circ}$ from the crystallographic $a$ and $b$ axes.

the magnetic anisotropy of fluorides of the iron group elements [5]. Early theoretical studies of the static magnetic properties of $\mathrm{CoF}_{2}$ in the paramagnetic and antiferromagnetic phases using an effective spin $S=3 / 2$ Hamiltonian were performed by Nakamura and Taketa [6]. The magnetic excitations in $\mathrm{CoF}_{2}$ were first studied by Lines [7] and the dominant exchange and anisotropy parameters were estimated by comparing theory with low-temperature antiferromagnetic resonance frequencies [8] and infrared absorption data [9]. The electronic stucture of the $\mathrm{Co}^{2+}$ ions has been determined by Gladney [10], and the spin-wave dispersion relations have been measured by Cowley et al. $[11,12]$ using inelastic neutron scattering. Raman light scattering measurements made by Macfarlane [13], Moch et al. [14], and Hoff et al. [15] have also characterized the low-lying zone center excitations, and the light scattering cross sections $[13,14]$ are somewhat consistent with the calculations of Ishikawa and Moriya [16]. The splitting of the excitations by an applied magnetic field has been studied in the infrared [17,18] and with Raman scattering [14].

In this paper we report experimental Raman scattering data for the one-magnon and exciton excitations in $\mathrm{CoF}_{2}$, together with a theoretical analysis for the magnetic excitations in a two-sublattice $S=3 / 2$ antiferromagnet with single-ion anisotropy. The theoretical technique used here employs the spin operators directly and allows us to investigate the magnetic excitations from the approximate ground state as well as the additional optical magnetic modes that are expected in this system.

The justification for using an effective spin $S=3 / 2$ model to study the low-lying magnetic excitations in $\mathrm{CoF}_{2}$ has been discussed by several authors $[6,7,16]$ and here we highlight the main arguments. The free $\mathrm{Co}^{2+}$ ion has an electronic configuration $3 d^{7}$ and application of Hund's rules yields a 28 -fold degenerate ${ }^{4} \mathrm{~F}$ ground state $(L=3$, $S=3 / 2$ ). When the ion is inserted into a crystal and surrounded by $\mathrm{F}^{-}$ions it is subjected to a perturbing electric field which lifts the degeneracy of the free ion. As the symmetry is lowered there is an increase in the number of split levels produced by the crystal field. The degeneracy of the orbital state of the free $\mathrm{Co}^{2+}$ ion is seven $(L=3)$ and is split into two triplets and a singlet by a crystalline electric field of cubic symmetry, where the lowest level is a triplet which is described in terms of an effective $L=1$ operator. The lowest orbital triplet state is now of degeneracy twelve because of the spin $S=3 / 2$ of the free ion. The $\mathrm{F}^{-}$ions surrounding the $\mathrm{Co}^{2+}$ ions do not have perfect cubic symmetry. The distortion from cubic symmetry combined with spin-orbit coupling splits the lowest energy manifold into six (Kramers) doublets. The degeneracy of each doublet is eventually removed by the exchange field, resulting in four lowest energy levels for the $\mathrm{Co}^{2+}$ ions $[10,16]$. The energies of these four levels (relative to the lowest) have been estimated $[10,16]$ to correspond roughly to $0,51,190$, and $200 \mathrm{~cm}^{-1}$, and they are well separated from the next level at about $800 \mathrm{~cm}^{-1}$.

This paper is organized as follows. In Sec. 2 we describe the Raman experiments and present the results for the one-magnon and exciton scattering in the $\mathrm{CoF}_{2}$ sample. The theoretical analyses for the magnon excitations are described in Sec. 3, where we mainly use the spin $S=3 / 2$ model which is superior to the effective spin $S=1 / 2$ approach in the context of the one-magnon and exciton Raman scattering. This contrasts with the situation for onemagnon light scattering in $\mathrm{NiF}_{2}$ mentioned earlier, where there the spin canting is important and the $\mathrm{Ni}^{2+}$ ions have spin $S=1$. Comparisons of the one-magnon theory and experimental data are presented in Sec. 4, and the other magnetic excitations are then briefly discussed in Sec. 5 . The conclusions of our work are given in Sec. 6 .

\section{Experiment and results}

The purplish-red-colored sample of $\mathrm{CoF}_{2}$ was prepared from a single crystal grown at the Clarendon Laboratory, Oxford University, specially for these one-magnon studies and our earlier two-magnon studies [1]. The cuboid sample of dimensions $3.2 \mathrm{~mm} \times 2.0 \mathrm{~mm} \times 1.7 \mathrm{~mm}$ was cut to expose (001) [Z], (110) $[X]$, and (110) $[Y]$ faces, respectively, and these faces were highly polished with $1 \mu \mathrm{m}$ diamond powder. The Raman spectrum was excited with 500-600 $\mathrm{mW}$ of Ti: sapphire laser light at $800 \mathrm{~nm}$, which avoided any optical absorption [19], analyzed with a Spex 14018 double monochromator at a spectral resolution of $3.3 \mathrm{~cm}^{-1}$ unless otherwise indicated, and detected by a cooled RCA 31034A photomultiplier. The sample was mounted in the helium exchange-gas space of a Thor S500 continuous flow cryostat, where the temperature could be controlled to 

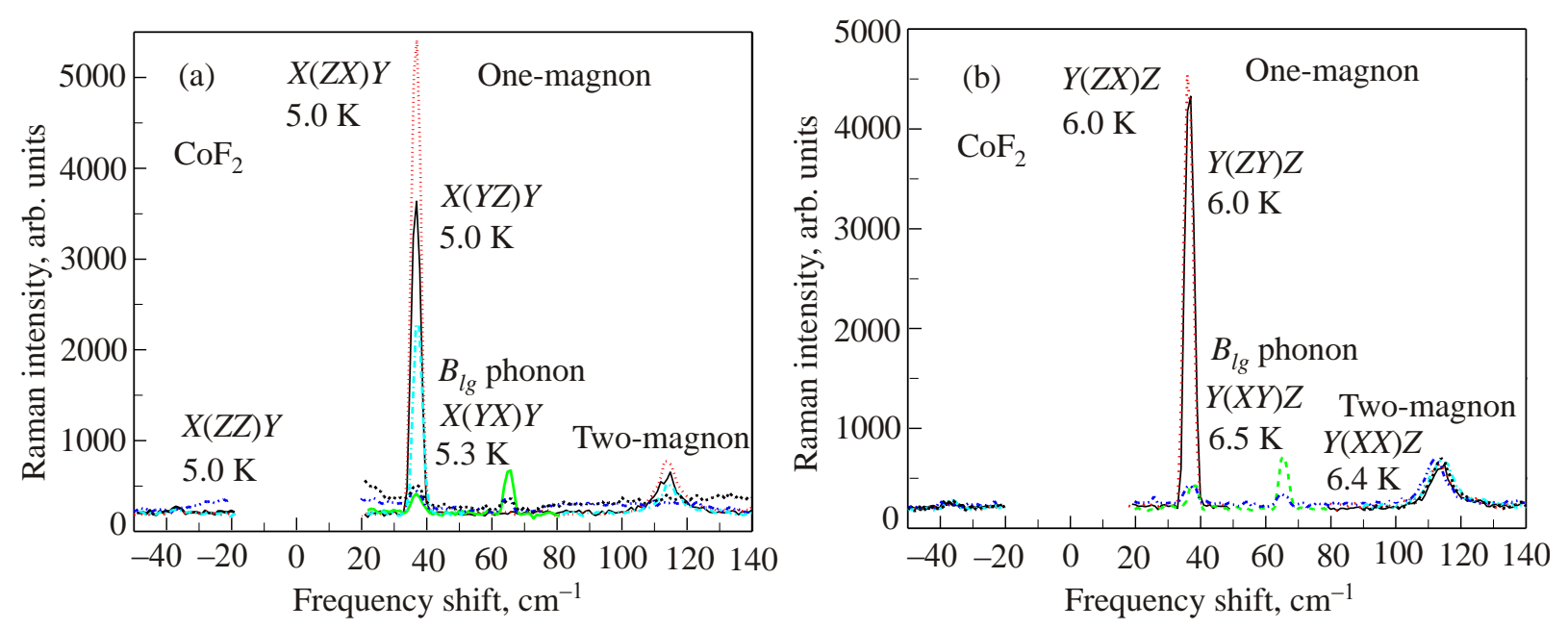

Fig. 2. (Color online) Polarization dependence of the low frequency Raman spectrum of antiferromagnetic $\mathrm{CoF}_{2}$ recorded at low temperature for $X(\cdots) Y\left(\right.$ a) and $Y(\cdots) Z$ (b) $90^{\circ}$ scattering geometries.

within $0.1 \mathrm{~K}$ and was measured with a gold-iron/chromel thermocouple clamped to the sample. Spectra were recorded in the $90^{\circ}$ scattering geometry. The one-magnon scattering was measured in different polarizations for temperatures up to about $T_{N}$, while the exciton features were investigated at low temperatures only.

The polarization dependence of the low frequency Raman spectrum of antiferromagnetic $\mathrm{CoF}_{2}$ at low temperature is shown in Fig. 2. These spectra exhibit a sharp peak at $(37.0 \pm 0.1) \mathrm{cm}^{-1}$ that is the lowest lying exciton (conventionally referred to as the magnon) of the ground state multiplet of the $\mathrm{Co}^{2+}$ ion in the exchange field. The one-magnon scattering is observed only in off-diagonal polarizations. The temperature dependences of the spectra for these same polarizations are given in Fig. 3, where it can be seen that the one-magnon peak decreases in frequency and increases in width with increasing temperature up to $T_{N}$ while its peak intensity decreases. It is evident that the anti-Stokes and Stokes intensities are also temperature dependent and vary with the polarization.
The Raman spectrum at higher frequencies exhibits three more sharp peaks associated with the higher lying excitons of the lowest multiplet, as shown in Fig. 4. These and other spectra were fitted with a GaussianLorentzian line shape model [20] to yield the band parameters of position, width (full width at half maximum), and integrated intensity. The results obtained at low temperature are given for the four excitons and also some $\mathrm{CoF}_{2}$ phonons in Table 1. This table shows that the widths of the lowest frequency exciton and phonon are resolution $\left(2.5 \mathrm{~cm}^{-1}\right)$ limited at low temperature. The phonon scattering is much stronger, in general, than the exciton scattering and the measurements in $X(Y Z) Y$ and $X(Z X) Y$ polarization give the expected similar intensities for the $E_{g}$ phonon, indicating that the experimental conditions are satisfactory, and this is also the case for exciton 3 , whereas for the magnon (exciton 1) they are different. The spectra shown in Fig. 4 indicate that the polarization leak through (e.g., of exciton 3 from $X(Y Z) Y$ polarization into $X(Z Z) Y$ polarization) is about $3 \%$.

Table 1. Band parameters of excitons and phonons obtained by curve fitting the polarized Stokes Raman spectra of $\mathrm{CoF}_{2}$ at $10 \mathrm{~K}$, as shown for the excitons in Fig. 4. The standard errors from the fits are given in brackets; FWHM is the band full width at half maximum.

\begin{tabular}{c|c|c|c|c}
\hline \hline Exciton/phonon number (symmetry) & Polarization & Frequency, cm & ${\text { FWHM, } \mathrm{cm}^{-1}}^{\text {Area, arb. units }}$ \\
\hline \hline $1\left(\Gamma_{3}^{+}+\Gamma_{4}^{+}\right)$ & $X(Y Z) Y$ & $36.9(0.1)$ & $2.6(0.1)$ & $1211(99)$ \\
$1\left(\Gamma_{3}^{+}+\Gamma_{4}^{+}\right)$ & $X(Z X) Y$ & $37.1(0.1)$ & $2.8(0.1)$ & $1853(163)$ \\
$2\left(\Gamma_{1}^{+}\right)$ & $X(Z Z) Y$ & $168.0(0.1)$ & $3.2(0.1)$ & $5479(157)$ \\
$3\left(\Gamma_{3}^{+}+\Gamma_{4}^{+}\right)$ & $X(Y Z) Y$ & $192.9(0.1)$ & $3.7(0.2)$ & $6118(570)$ \\
$3\left(\Gamma_{3}^{+}+\Gamma_{4}^{+}\right)$ & $X(Z X) Y$ & $193.0(0.1)$ & $3.6(0.2)$ & $7294(557)$ \\
$4\left(\Gamma_{2}^{+}\right)$ & $X(Y X) Y$ & $209.1(0.4)$ & $4.0(1.1)$ & $142(85)$ \\
$1\left(B_{1 g}\right)$ & $X(Y X) Y$ & $65.2(0.1)$ & $2.3(0.2)$ & $224(47)$ \\
$2\left(E_{g}\right)$ & $X(Y Z) Y$ & $255.3(0.1)$ & $4.7(0.1)$ & $30837(1884)$ \\
$2\left(E_{g}\right)$ & $X(Z X) Y$ & $255.3(0.1)$ & $4.7(0.1)$ & $29374(1317)$ \\
$3\left(A_{1 g}\right)$ & $X(Z Z) Y$ & $371.2(0.1)$ & $3.8(0.1)$ & $20290(76)$ \\
\hline \hline
\end{tabular}



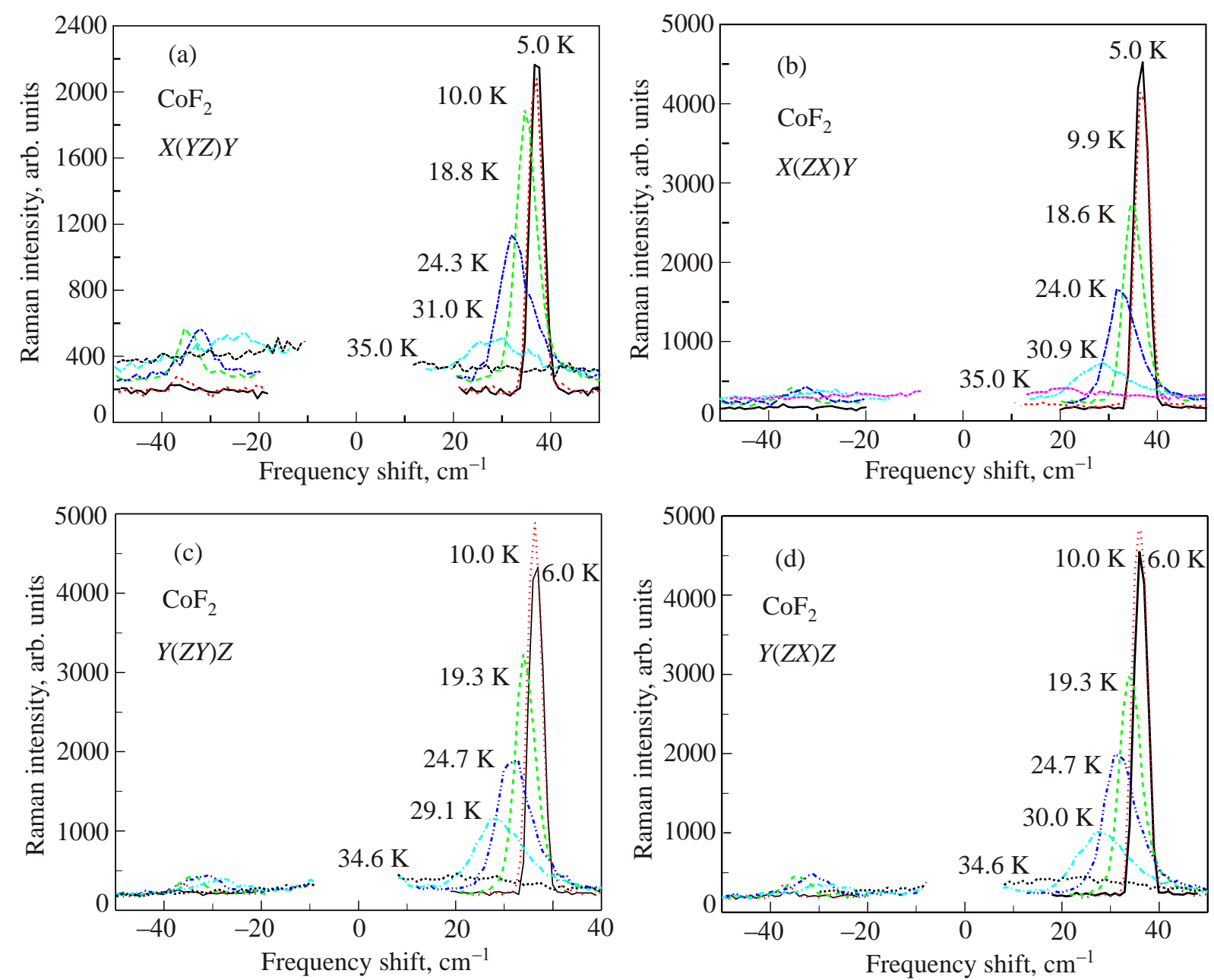

Fig. 3. (Color online) Temperature dependence of the Stokes and anti-Stokes one-magnon Raman spectrum of antiferromagnetic $\mathrm{CoF}_{2}$ for $X(Y Z) Y($ a) $, X(Z X) Y(b), Y(Z Y) Z(\mathrm{c})$, and $Y(Z X) Z(\mathrm{~d})$ polarization.

Our measurements for the frequencies of the four lowestlying excitons are compared with earlier results in Table 2, and there is good agreement between values obtained by Raman scattering and also the quite different techniques of inelastic neutron scattering and infrared spectroscopy. This is not the case, however, when it comes to the Raman crosssections of the excitons. As can be seen from Table 3, the integrated intensities of the excitons observed in the various polarizations when normalized to that found for exciton 3 in $X(Y Z) Y$ polarization can be quite different for the three experimental studies reported to date. In addition, there is a greater discrepancy between our values compared with previous theory than for the other two experimental studies. However, there is one point on which all experiments and theory agree and that is the relative integrated intensity of exciton 4 is much weaker than for the others. It is evident

Table 2. Comparison of results obtained in different experiments and from theory for the lower multiplet exciton frequencies (in $\mathrm{cm}^{-1}$ ) in $\mathrm{CoF}_{2}$ at low temperature $\left(T \ll T_{N}\right)$.

\begin{tabular}{c|c|c|c|c|c|c}
\hline $\begin{array}{c}\text { Exciton number } \\
\text { (symmetry) }\end{array}$ & $\begin{array}{c}\text { Raman } \\
\text { Present work }\end{array}$ & $\begin{array}{c}\text { Raman } \\
\text { Ref. 13 }\end{array}$ & $\begin{array}{c}\text { Raman } \\
\text { Ref. 14 }\end{array}$ & $\begin{array}{c}\text { Infrared } \\
\text { Ref. 18 }\end{array}$ & $\begin{array}{c}\text { Neutron Scattering } \\
\text { Ref. 12 }\end{array}$ & $\begin{array}{c}\text { Theory } \\
\text { Ref. 16 }\end{array}$ \\
\hline $1\left(\Gamma_{3}^{+}+\Gamma_{4}^{+}\right)$ & $37.0 \pm 0.1$ & $37.0 \pm 0.5$ & 37 & 36.3 & 37 & 37 \\
$2\left(\Gamma_{1}^{+}\right)$ & $168.0 \pm 0.1$ & 169 & 168.3 & 168.5 & 170 & 173 \\
$3\left(\Gamma_{3}^{+}+\Gamma_{4}^{+}\right)$ & $193.0 \pm 0.1$ & 194 & 193.3 & 193.0 & 196 & 200 \\
$4\left(\Gamma_{2}^{+}\right)$ & $209.0 \pm 0.1$ & - & 210.2 & $210.9^{*}$ & 204 & 206 \\
\hline \hline
\end{tabular}

Notes: *As deduced by Moch et al. [14] from the magnetic field results in the infrared study of Ref. 18. 

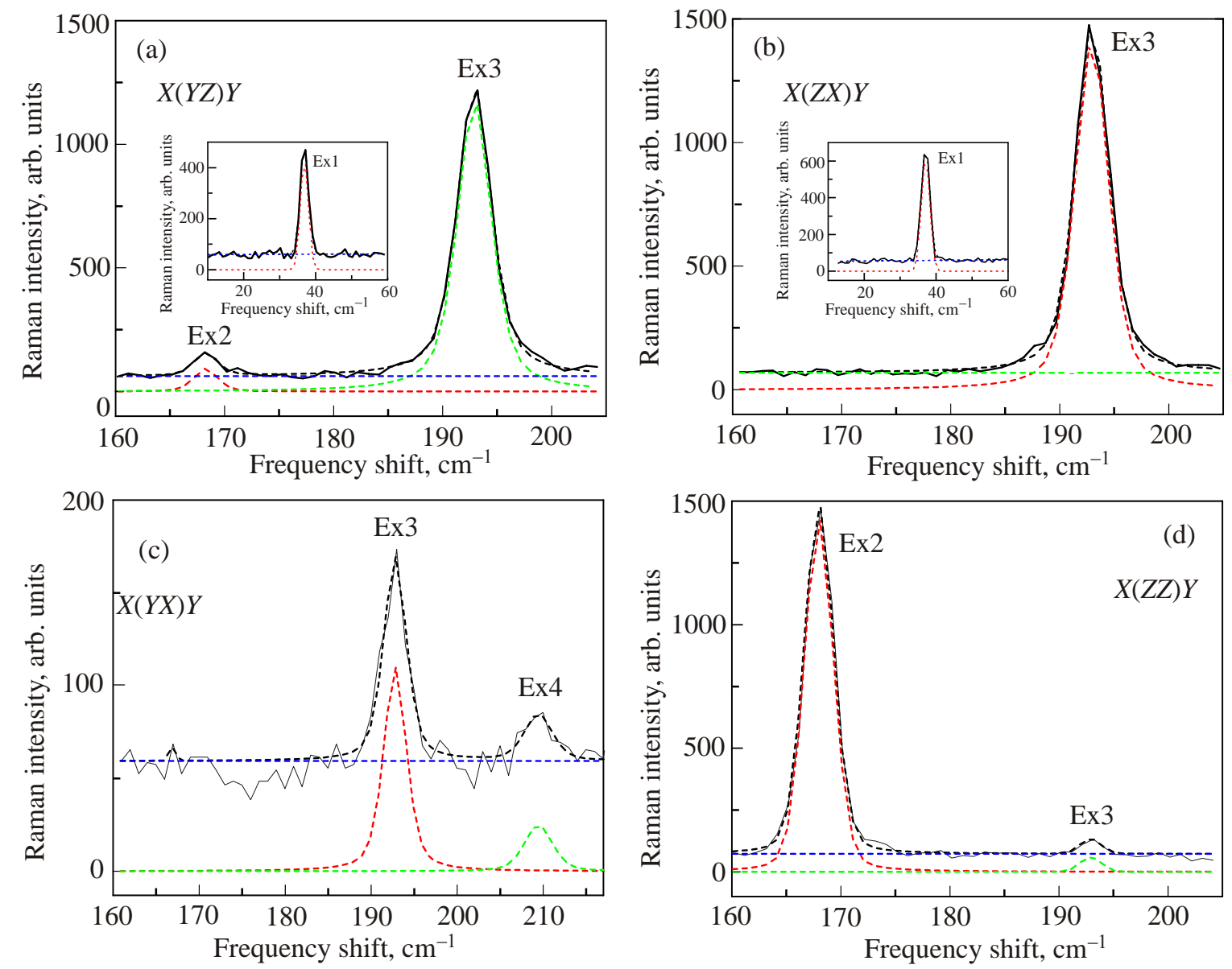

Fig. 4. (Color online) Raman spectra of the lowest energy excitons in antiferromagnetic $\mathrm{CoF}_{2}$ at $10 \mathrm{~K}$ and their line shape fits with the Gaussian-Lorentzian model in $X(Y Z) Y(\mathrm{a}), X(Z X) Y(\mathrm{~b}), X(Y X) Y(\mathrm{c})$, and $X(Z Z) Y(\mathrm{~d})$ polarization. The spectral resolution for these spectra was $2.5 \mathrm{~cm}^{-1}$.

from the theoretical data given in Table 3 that the excitation energy is a crucial factor in elaborating the Raman cross section, presumably because of possible Raman excitation energy resonances with higher-lying electronic energy levels of the $\mathrm{Co}^{2+}$ ion. The experimental and theoretical data indicate that there is an increase in the overall intensities, rela- tive to the fixed (reference) one, of the excitons in the other polarizations with Raman excitation energy increase over the range 12500 to $30000 \mathrm{~cm}^{-1}$. At the same time, theory shows that there is a large absolute intensity increase when the excitation energy is changed from 20000 to $30000 \mathrm{~cm}^{-1}$ (see Table 3).

Table 3. Comparison of results obtained in different experiments and from theory at different excitation energies $\left(v\right.$ in $\left.\mathrm{cm}^{-1}\right)$ for the lower multiplet exciton relative Raman intensities in $\mathrm{CoF}_{2}$ at low temperature $\left(T \ll T_{N}\right)$.

\begin{tabular}{c|c|c|c|c|c|c|c}
\hline $\begin{array}{c}\text { Exciton number } \\
\text { (symmetry) }\end{array}$ & Polarization & $\begin{array}{c}\text { Experiment } \\
\text { Present Work } \\
v=12500\end{array}$ & $\begin{array}{c}\text { Experiment } \\
\text { Ref. 13 } \\
v=16000\end{array}$ & $\begin{array}{c}\text { Experiment } \\
\text { Ref. 14 } \\
v=16000\end{array}$ & $\begin{array}{c}\text { Theory } \\
\text { Ref. 13* }\end{array}$ & $\begin{array}{c}\text { Theory } \\
\text { Ref. } 16 \\
v=20000\end{array}$ & $\begin{array}{c}\text { Theory } \\
\text { Ref. } 16 \\
v=30000\end{array}$ \\
\hline \hline $1\left(\Gamma_{3}^{+}+\Gamma_{4}^{+}\right)$ & $X(Y Z) Y$ & $20 \pm 4$ & 28 & $29 \pm 6$ & 36 & 50 & 86 \\
& $X(Z X) Y$ & $30 \pm 6$ & 27 & $60 \pm 12$ & 79 & 125 & 180 \\
$2\left(\Gamma_{1}^{+}\right)$ & $Y(X X) Z$ & - & $<1.3$ & $22 \pm 4$ & 200 & 225 & 225 \\
$\left.3\left(\Gamma_{3}^{+}+\Gamma_{4}^{+}\right)\right)$ & $X(Z Z) Y$ & $90 \pm 11$ & 65 & $220 \pm 44$ & 340 & 375 & 380 \\
& $X(Y Z) Y$ & 100 & 100 & 100 & 100 & 100 & $100^{\star *}$ \\
$4\left(\Gamma_{2}^{+}\right)$ & $X(Z X) Y$ & $120 \pm 20$ & 110 & $130 \pm 26$ & 180 & 200 & 250 \\
\hline \hline
\end{tabular}

Notes: * Ref. 13 quotes unpublished theoretical work of A. Ishikawa and T. Moriya.

${ }^{* *}$ In absolute terms, the theoretical intensity for this band with $v=30000$ is 5.5 times that of the $v=20000$ case. 
The one-magnon spectra as a function of temperature and polarization were all readily fitted with a GaussianLorentzian line shape model and the results obtained for the line parameters in Stokes and anti-Stokes scattering are given in Figs. 5 and 6, respectively. For comparison with theory, we also give in Fig. 7 the temperature de-
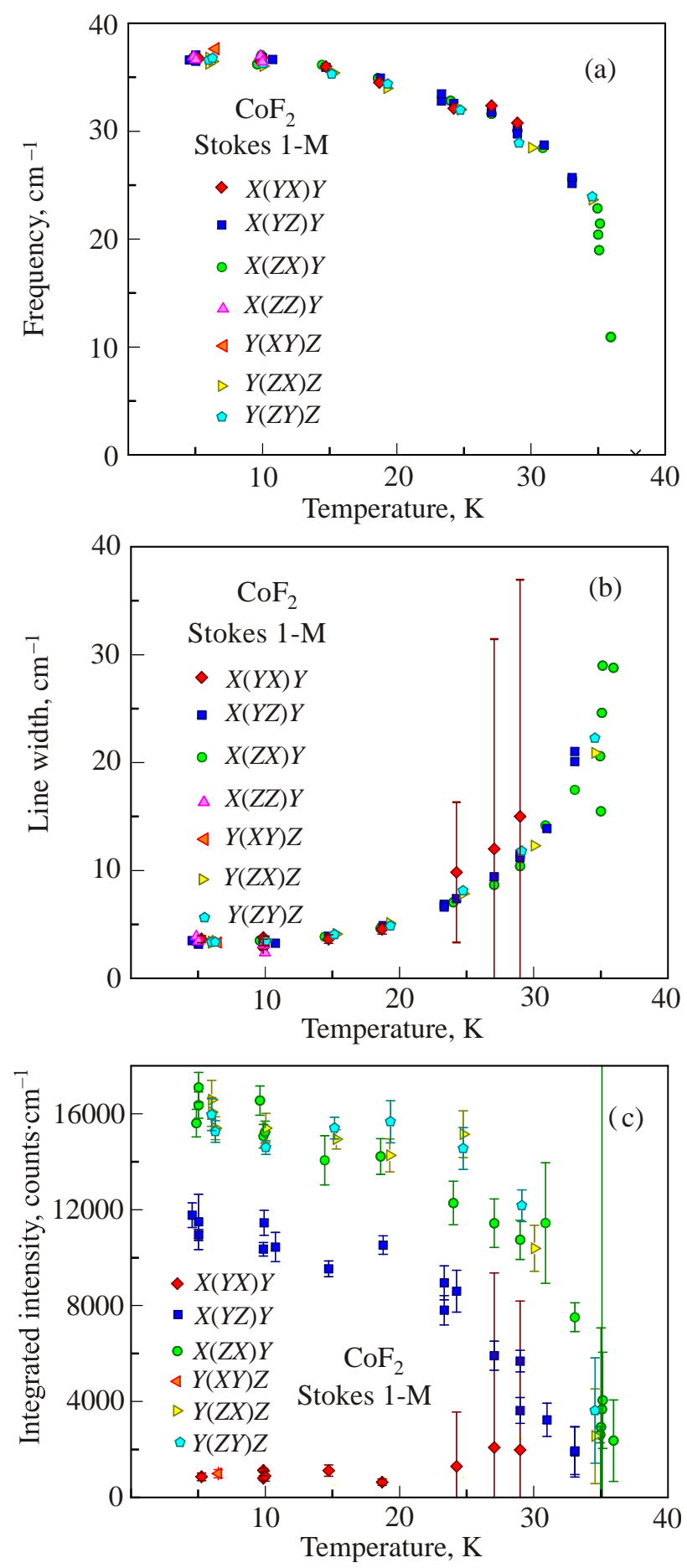

Fig. 5. (Color online) Temperature dependence of the $\mathrm{CoF}_{2}$ Stokes one-magnon Raman scattering fitted line shape parameters of frequency (a), full width at half maximum (b), and integrated intensity (c) for the various polarizations indicated. pendences of the ratio of the Stokes to anti-Stokes onemagnon integrated intensities for several polarizations. The intensity ratios for all polarizations exhibit a similar steep rise with decreasing temperature below about $15 \mathrm{~K}$. The results in $X(Y Z) Y$ polarization, although showing a different temperature dependence from the other polariza-
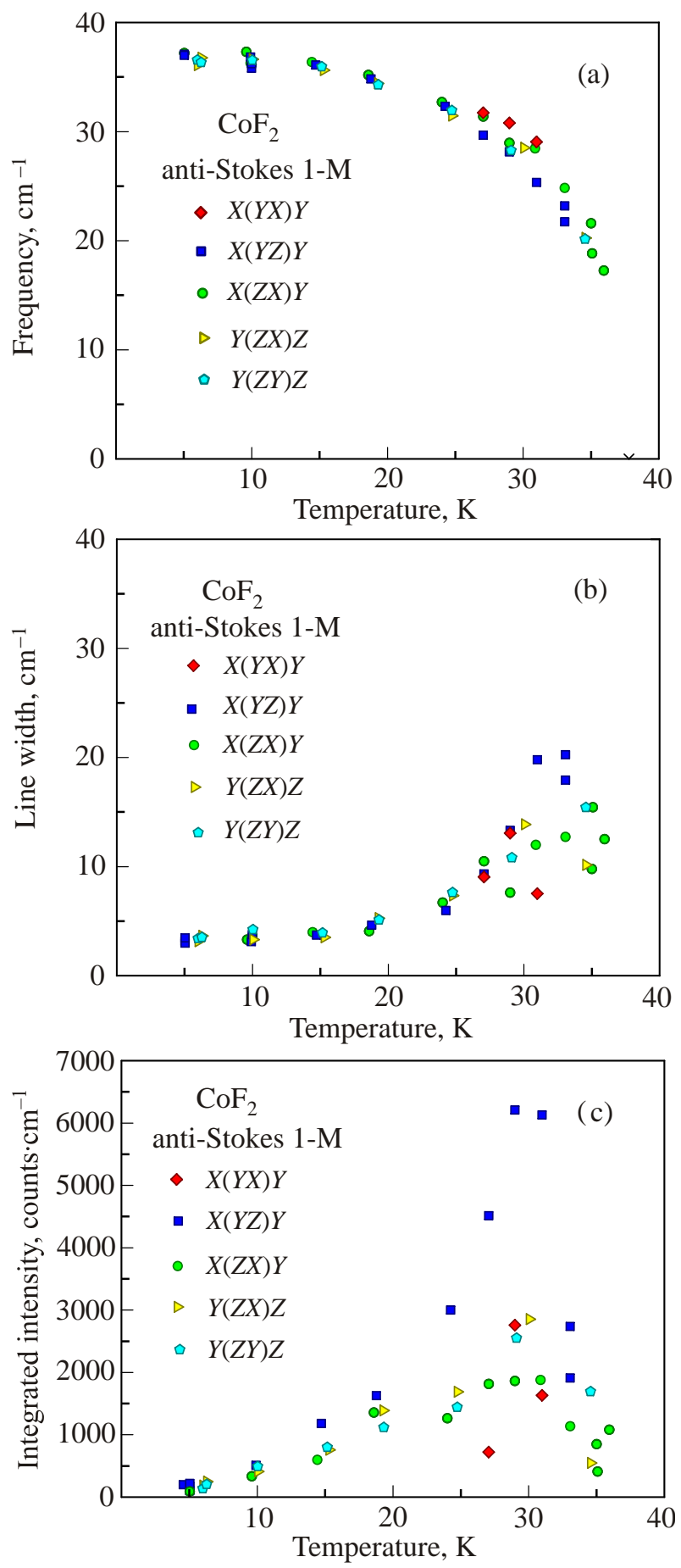

Fig. 6. (Color online) Temperature dependence of the $\mathrm{CoF}_{2}$ antiStokes one-magnon Raman scattering fitted line shape parameters of frequency (a), full width at half maximum (b), and integrated intensity (c) for the various polarizations indicated. 


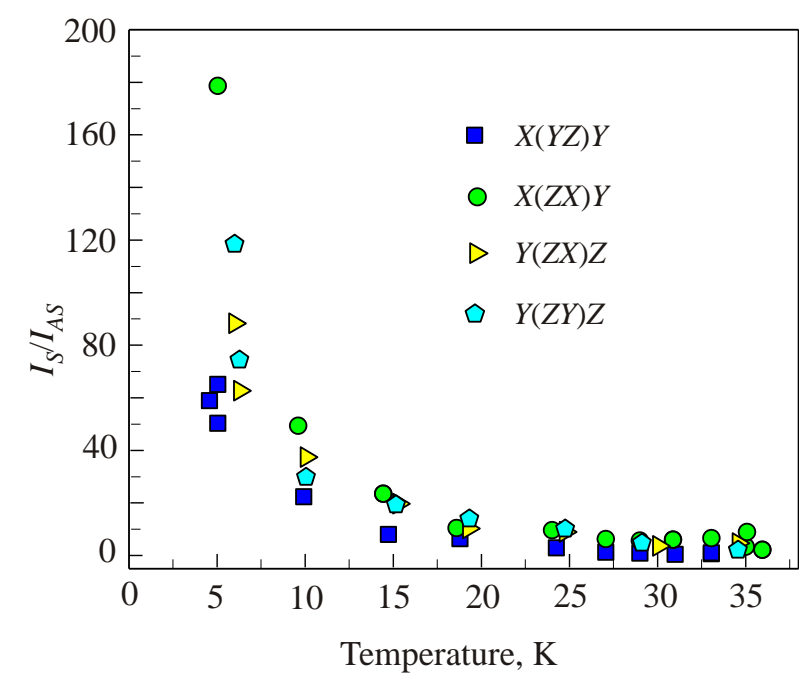

Fig. 7. Temperature dependence of the ratio of the $\mathrm{CoF}_{2}$ Stokes $\left(I_{S}\right)$ and anti-Stokes $\left(I_{A S}\right)$ one-magnon integrated intensities in $X(Y Z) Y, \quad X(Z X) Y, \quad Y(Z X) Z$, and $Y(Z Y) Z$ polarization.

tions in Figs. 5(c) and 6(c), behave similarly to the others in the intensity ratio variation with temperature.

\section{One-magnon theoretical analysis}

For simplicity we start by briefly presenting an effective spin $S=1 / 2$ Green's function theory for the onemagnon excitations. This approach is useful when analyzing two-magnon Raman scattering [1] because approximations appropriate to large wave vectors can be made, but it has its limitations for magnons near the Brillouin-zone center. Therefore it is followed by an effective $S=3 / 2$ theory, which is expected to provide a more realistic descriptions of the magnons since it takes into account the lowest four levels (instead of just two). In particular, the latter approach is expected to account more accurately for anisotropy effects and to be superior at elevated temperatures.

\subsection{Effective spin $1 / 2$ theory}

This model [7] is based on taking account of only the lowest two energy levels for the $\mathrm{Co}^{2+}$ ions, which are separated by about $50 \mathrm{~cm}^{-1}$ as already noted. The effective Hamiltonian can be expressed as

$$
\mathcal{H}=\mathcal{H}_{\mathrm{ex}}+\mathcal{H}_{\mathrm{an}}
$$

where $\mathcal{H}_{\text {ex }}$ and $\mathcal{H}_{\text {an }}$ refer to the exchange and anisotropy parts respectively, with

$$
\begin{gathered}
\mathcal{H}_{\mathrm{ex}}=\sum_{i, j} J_{i, j} \mathbf{S}_{i} \cdot \mathbf{S}_{j}+\frac{1}{2} \sum_{i, i^{\prime}} J_{i, i^{\prime}}^{\prime} \mathbf{S}_{i} \cdot \mathbf{S}_{i^{\prime}}+\frac{1}{2} \sum_{j, j^{\prime}} J_{j, j^{\prime}}^{\prime} \mathbf{S}_{i} \cdot \mathbf{S}_{j^{\prime}} \\
\mathcal{H}_{\mathrm{an}}=-H_{A}(T)\left(\sum_{i} S_{i}^{z}-\sum_{j} S_{j}^{z}\right)
\end{gathered}
$$

The sites labelled $i$ are on the spin-up sublattice and those labelled $j$ are on the spin-down sublattice. The intersublattice exchange interaction is represented by $J_{i, j}$ whereas $J_{i, i^{\prime}}^{\prime}$ and $J_{j, j^{\prime}}^{\prime}$ are the intrasublattice exchanges. The effective anisotropy field $H_{A}(T)$ is often assumed to vary with temperature like the sublattice magnetization. The parameters for the $S=1 / 2$ models have been previously estimated by comparing theory with one-magnon inelastic neutron scattering measurements [11], yielding $J_{1}=-2.0$ $\mathrm{cm}^{-1}, J_{2}=12.3 \mathrm{~cm}^{-1}, J_{3} \sim 0 \mathrm{~cm}^{-1}$ and $H_{A}(0)=12.5 \mathrm{~cm}^{-1}$ where the exchange parameters are defined in Fig. 1.

The one-magnon excitation energies are obtained by forming the operator equations of motion for the $S^{+}$operators for each sublattice using the above Hamiltonian. The equations are then linearized using the random phase approximation (RPA) and transformed to a wave vector representation. The solutions for the excitation energies are obtained, assuming a time dependence $\exp (i E t)$ for the $S^{+}$operators, as [1]

$$
E_{\mathbf{k}}= \pm \sqrt{\mu^{2}(\mathbf{k})-\left(8\left\langle S^{z}\right\rangle J_{2} \cos \left(k_{x} a / 2\right) \cos \left(k_{y} a / 2\right) \cos \left(k_{z} c / 2\right)\right)^{2}}
$$

where

$$
\mu(\mathbf{k})=H_{A}(T)+8\left\langle S^{z}\right\rangle J_{2}-4\left\langle S^{z}\right\rangle J_{1} \sin ^{2}\left(k_{z} c / 2\right)-4\left\langle S^{z}\right\rangle J_{3}\left[\sin ^{2}\left(k_{x} a / 2\right)+\sin ^{2}\left(k_{y} a / 2\right)\right] .
$$

The energies are degenerate in magnitude (in the zero applied field case considered here) and the negative sign in Eq. (4) refers to oppositely precessing spins. The spin average $\left\langle S^{z}\right\rangle$ related to the sublattice magnetization can be evaluated using mean-field theory [1].

\subsection{Effective spin $3 / 2$ theory}

Next we focus on the $S=3 / 2$ theory obtained using the four lowest energy levels for the $\mathrm{Co}^{2+}$ ions [16]. This is expected to be superior here because of the more careful treatment of the anisotropy, which is particularly important for one-magnon Raman scattering. The two approaches will be compared later. In this case the total Hamiltonian can again be expressed as in Eq. (1) except that all spin operators now refer to $S=3 / 2$ and the anisotropy part is replaced by

$$
\begin{gathered}
\mathcal{H}_{\mathrm{an}}=\sum_{i}\left(D\left(S_{i}^{z}\right)^{2}+F\left[\left(S_{i}^{x}\right)^{2}-\left(S_{i}^{y}\right)^{2}\right]\right)+ \\
+\sum_{j}\left(D\left(S_{j}^{z}\right)^{2}-F\left[\left(S_{j}^{x}\right)^{2}-\left(S_{j}^{y}\right)^{2}\right]\right) .
\end{gathered}
$$


Here the parameters $D$ and $F$ describe the effects of the uniaxial and nonuniaxial contributions to the single-ion anisotropy, respectively. The average spin alignment for each sublattice is assumed to be along the crystallographic $c$ axis as depicted schematically in Fig. 1 .

We now use the Green's function equation of motion method (rather than the operator equation of motion, which provides less information) to generate the set of equations satisfied by $\ll S_{i}^{+} ; Y \gg_{E}$ and the other Green's functions coupled to it. As before we use RPA decoupling for products of spins at different sites, but we do not approximate the anisotropy terms which involve products of operators at the same site. The formalism is analogous to that employed by Cottam and Latiff Awang [21] for $S=1$ antiferromagnets with single-ion anisotropy, but extended here to $S=3 / 2$. To obtain a finite, closed set of equations, we use the identity for $S=3 / 2$ spin operators that $\left(S^{z}\right)^{4}=(5 / 2)\left(S^{z}\right)^{2}-(9 / 16)$. Taking into account the different sublattice labeling, 16 coupled equations are required to obtained a closed set of equations. After a transformation to wave vector representation the set of equations can be written in matrix form as

$$
\left(E \mathbf{I}_{16}+\mathbf{B}\right) \mathbf{G}_{\mathbf{k}}=\mathbf{b},
$$

where $\mathbf{I}_{16}$ is the $16 \times 16$ unit matrix, $\mathbf{G}_{\mathbf{k}}$ and $\mathbf{b}$ are 16component column matrices whose elements are defined as $G_{n \mathbf{k}}$ and $b_{n}($ for $n=1, \ldots, 16)$ with

$$
\begin{gathered}
\ll X_{n} ; Y \gg_{E}=\frac{1}{N} \sum_{\mathbf{k}} \exp \left[i \mathbf{k} \cdot\left(\mathbf{r}_{l}-\mathbf{r}_{m}\right)\right] G_{n \mathbf{k}}, \\
b_{n}=\frac{1}{N}\left\langle\left[X_{n}, Y\right]\right\rangle,
\end{gathered}
$$

for any operator $Y$. We define the operators $X_{n}$ as

$$
\begin{gathered}
X_{1}=S_{i}^{+}, \quad X_{2}=S_{j}^{+}, \quad X_{3}=S_{i}^{-}, \quad X_{4}=S_{j}^{-}, \\
X_{5}=S_{i}^{+} S_{i}^{z}+S_{i}^{z} S_{i}^{+}, \quad X_{6}=S_{j}^{+} S_{j}^{z}+S_{j}^{z} S_{j}^{+}, \\
X_{7}=S_{i}^{-} S_{i}^{z}+S_{i}^{z} S_{i}^{-}, \quad X_{8}=S_{j}^{-} S_{j}^{z}+S_{j}^{z} S_{j}^{-}, \\
X_{9}=\left(S_{i}^{z}\right)^{2} S_{i}^{+}+2 S_{i}^{z} S_{i}^{+} S_{i}^{z}+S_{i}^{+}\left(S_{i}^{z}\right)^{2}, \\
X_{10}=\left(S_{j}^{z}\right)^{2} S_{j}^{+}+2 S_{j}^{z} S_{j}^{+} S_{j}^{z}+S_{j}^{+}\left(S_{j}^{z}\right)^{2}, \\
X_{11}=\left(S_{i}^{z}\right)^{2} S_{i}^{-}+2 S_{i}^{z} S_{i}^{-} S_{i}^{z}+S_{i}^{-}\left(S_{i}^{z}\right)^{2}, \\
X_{12}=\left(S_{j}^{z}\right)^{2} S_{j}^{-}+2 S_{j}^{z} S_{j}^{-} S_{j}^{z}+S_{j}^{-}\left(S_{j}^{z}\right)^{2}, \\
X_{13}=\left(S_{i}^{+}\right)^{3}, \quad X_{14}=\left(S_{j}^{+}\right)^{3}, \\
X_{15}=\left(S_{i}^{-}\right)^{3}, \quad X_{16}=\left(S_{j}^{-}\right)^{3} .
\end{gathered}
$$

The above operators $X_{1}, \ldots, X_{12}$ are all linear in the transverse spin components and are associated with transi- tions for $\Delta S^{z}= \pm 1$ whereas the operators $X_{13}, \ldots, X_{16}$ all involve transitions for $\Delta S^{z}= \pm 3$. We note that these coupled equations do not involve any operators in combinations that are quadratic in the transverse spin components. The excitations associated with this combination of spin operators are different from the one-magnon excitations and so are considered in a later section.

By comparison with the case of an anisotropic $S=1$ antiferromagnet considered earlier [21] using this operator method, the analogous equation to Eq. (7) involved only a $8 \times 8$ matrix, because the operator combinations $X_{9}, \ldots, X_{16}$ in that case can be shown either to be zero or to be expressible in terms of the remaining $X_{1}, \ldots, X_{8}$. In general, as $S$ is increased, it is found that more coupled equations of motion are needed to obtain a closed set.

In our analysis the poles of the Green functions correspond to the spin-wave (one-magnon) excitations, which are obtained by applying the determinantal condition that

$$
\operatorname{det}\left(E \mathbf{I}_{16}+\mathbf{B}\right)=0 \text {, }
$$

as follows from Eq. (7). From the formal results for Green's functions we can also deduce the spin correlation functions by means of the fluctuation-dissipation theorem and thus evaluate light scattering cross sections [21]. The nonzero elements of the matrix $\mathbf{B}$ are listed in the Appendix. The expressions involve wave-vector Fourier transforms of exchange terms defined by

$$
\begin{gathered}
J_{1}(\mathbf{k})=2 J_{1} \cos \left(k_{z} c\right), \\
J_{2}(\mathbf{k})=8 J_{2} \cos \left(k_{x} a / 2\right) \cos \left(k_{y} a / 2\right) \cos \left(k_{z} c / 2\right), \\
J_{3}(\mathbf{k})=2 J_{3}\left(\cos \left(k_{x} a\right)+\cos \left(k_{y} a\right)\right),
\end{gathered}
$$

and single-site thermal averages (on the spin-up sublattice) corresponding to

$$
\begin{gathered}
m_{1}=\left\langle S_{i}^{z}\right\rangle, \quad m_{2}=3\left\langle\left(S_{i}^{z}\right)^{2}\right\rangle-15 / 4, \\
m_{3}=\left\langle\left(S_{i}^{+}\right)^{2} S_{i}^{z}+2 S_{i}^{+} S_{i}^{z} S_{i}^{+}+S_{i}^{z}\left(S_{i}^{+}\right)^{2}\right\rangle= \\
=\left\langle\left(S_{i}^{-}\right)^{2} S_{i}^{z}+2 S_{i}^{-} S_{i}^{z} S_{i}^{-}+S_{i}^{z}\left(S_{i}^{-}\right)^{2}\right\rangle, \\
m_{4}=8\left\langle\left(S_{i}^{z}\right)^{3}\right\rangle-14\left\langle S_{i}^{z}\right\rangle, \quad m_{5}=\left\langle\left(S_{i}^{+}\right)^{2}\right\rangle=\left\langle\left(S_{i}^{-}\right)^{2}\right\rangle .
\end{gathered}
$$

The above thermal averages can now be estimated using a modified mean-field theory.

\subsection{Thermal averages}

We use the standard quantum-mechanical representation for the spin $S=3 / 2$ operators in terms of $4 \times 4$ matrices:

$$
S^{z}=\left(\begin{array}{cccc}
3 / 2 & 0 & 0 & 0 \\
0 & 1 / 2 & 0 & 0 \\
0 & 0 & -1 / 2 & 0 \\
0 & 0 & 0 & -3 / 2
\end{array}\right), \quad S^{+}=\left(\begin{array}{cccc}
0 & \sqrt{3} & 0 & 0 \\
0 & 0 & 2 & 0 \\
0 & 0 & 0 & \sqrt{3} \\
0 & 0 & 0 & 0
\end{array}\right) .
$$


Following the general approach as used for $S=1$ antiferromagnets [21], the thermal averages are calculated using an effective Hamiltonian where we adopt a mean-field approximation to simplify the exchange terms but treat the single-ion anisotropy terms exactly. The effective Hamiltonian for any site on the $i$-sublattice may be written as the matrix

$H_{\text {eff }}=\left(\begin{array}{cccc}-\frac{3}{2}\left(h_{0}-\frac{3}{2} D\right) & 0 & -\sqrt{3} F & 0 \\ 0 & -\frac{1}{2}\left(h_{0}-\frac{1}{2} D\right) & 0 & -\sqrt{3} F \\ -\sqrt{3} F & 0 & \frac{1}{2}\left(h_{0}+\frac{1}{2} D\right) & 0 \\ 0 & -\sqrt{3} F & 0 & \frac{3}{2}\left(h_{0}+\frac{3}{2} D\right)\end{array}\right)$

where $h_{0}=\left\langle S^{z}\right\rangle\left[J_{1}(0)+J_{2}(0)+J_{3}(0)\right]$ is an effective exchange field. The eigenvalues of Eq. (14) are found to be

$$
\begin{aligned}
& \lambda_{1,3}=\left(\frac{5 D}{4}-\frac{h_{0}}{2}\right) \mp \sqrt{\left(h_{0}-D\right)^{2}+3 F^{2}}, \\
& \lambda_{2,4}=\left(\frac{5 D}{4}+\frac{h_{0}}{2}\right) \mp \sqrt{\left(h_{0}+D\right)^{2}+3 F^{2}},
\end{aligned}
$$

and the eigenvectors are

$$
\begin{aligned}
& \left(\begin{array}{l}
\left|\lambda_{1}\right\rangle \\
\left|\lambda_{3}\right\rangle
\end{array}\right)=\frac{1}{\sqrt{\alpha^{2}+1}}\left(\begin{array}{cc}
\alpha & 1 \\
1 & -\alpha
\end{array}\right)\left(\begin{array}{c}
\left|\frac{3}{2}, \frac{3}{2}\right\rangle \\
\left|\frac{3}{2},-\frac{1}{2}\right\rangle
\end{array}\right), \\
& \left(\begin{array}{l}
\left|\lambda_{2}\right\rangle \\
\left|\lambda_{4}\right\rangle
\end{array}\right)=\frac{1}{\sqrt{\beta^{2}+1}}\left(\begin{array}{cc}
1 & \beta \\
-\beta & 1
\end{array}\right)\left(\begin{array}{c}
\left|\frac{3}{2}, \frac{1}{2}\right\rangle \\
\left|\frac{3}{2},-\frac{3}{2}\right\rangle
\end{array}\right),
\end{aligned}
$$

in a standard notation. Here we have defined the factors

$$
\begin{aligned}
& \alpha=\frac{-1}{\sqrt{3} F}\left\{\left(h_{0}-D\right)+\sqrt{\left(h_{0}-D\right)^{2}+3 F^{2}}\right\}, \\
& \beta=\frac{-1}{\sqrt{3} F}\left\{\left(h_{0}+D\right)-\sqrt{\left(h_{0}+D\right)^{2}+3 F^{2}}\right\} .
\end{aligned}
$$

As mentioned earlier, in the absence of exchange (i.e., if we set $h_{0}=0$ ) we would obtain just two sets of degenerate energy eigenvalues which represent two low-lying doublets. The separation between these doublets has been previously estimated $[7,10,16]$ to be within the range 152 to $175 \mathrm{~cm}^{-1}$. This, together with the data available from light scattering, allows an estimate to be made of the values of the anisotropy and exchange parameters of the spin Hamiltonian. Therefore in Fig. 8 we show a schematic of the energy level splitting produced by the exchange field. The operators $X_{1}, \ldots, X_{12}$ in Eq. (9) are all linear in a transverse spin component and thus correspond to the transitions labeled as $\Delta_{\alpha}, \Delta_{\beta}$, and

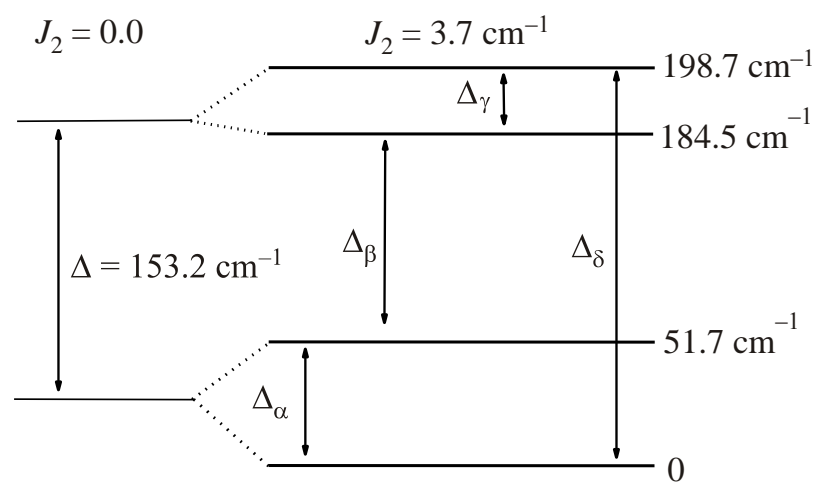

Fig. 8. Schematic representation of the four lowest energy levels of the $\mathrm{Co}^{2+}$ ions showing the effects of the exchange field. The relative energies are calculated using Eq. (15) and the parameters $J_{2}=3.7 \mathrm{~cm}^{-1}, D=-23.6 \mathrm{~cm}^{-1}$, and $F=-42.1 \mathrm{~cm}^{-1}$, ignoring the small effects of $J_{1}$ and $J_{3}$. The transitions (marked as $\Delta_{\alpha}, \Delta_{\beta}, \Delta_{\gamma}$, and $\Delta_{\delta}$ ), between these energy levels are discussed in the text.

$\Delta_{\gamma}$, whereas the operators $X_{13}, \ldots, X_{16}$ are cubic in a transverse spin component and correspond to the transition labeled as $\Delta_{\delta}$. These mean-field transition energies are different, in general, from the energies of the spin waves, because the latter include spin-fluctuation effects absent in mean-field theory. The energy level spacings indicated in Fig. 8 are calculated using Eq. (15) with the parameters $J_{2}=$ $=3.7 \mathrm{~cm}^{-1}, D=-23.6 \mathrm{~cm}^{-1}$ and $F=-42.1 \mathrm{~cm}^{-1}$. The value of the dominant antiferromagnet exchange term $J_{2}$ was chosen such that the energy of the lowest-lying $\mathbf{k}=0$ spin wave is $\approx 37 \mathrm{~cm}^{-1}$, in accordance with the light scattering data. We note that the fitted exchange parameters are expected to be different in the $S=3 / 2$ model because of the different role of the anisotropy terms and the different spin quantum number.

In the low-temperature limit $\left(T \ll T_{N}\right)$ the static thermal average for any operator $A$ is defined as $\left.<\lambda_{1}|A| \lambda_{1}\right\rangle$ where $\left|\lambda_{1}\right\rangle$ is the mean-field ground state eigenfunction. The corresponding spin thermal averages defined in Eq. (12) are then found to have the limiting $T=0$ values

$$
\begin{gathered}
m_{1}=\frac{1}{2 \sqrt{\alpha^{2}+1}}\left(3 \alpha^{2}-1\right), \quad m_{2}=\frac{3}{\sqrt{\alpha^{2}+1}}\left(\alpha^{2}-1\right), \\
m_{3}=2 m_{5}=4 \sqrt{3} \frac{\alpha}{\alpha^{2}+1}, \quad m_{4}=6 .
\end{gathered}
$$

At elevated temperatures the higher energy states of the four-level system become thermally populated and the static thermal averages must then be evaluated using

$$
\langle X\rangle=\sum_{i=1}^{4}\left\langle\lambda_{i}|X| \lambda_{i}>\exp \left(-\lambda_{i} / k_{B} T\right) .\right.
$$

This result is employed in Fig. 9 to calculate the temperature dependence (up to $T_{N}$ ) of the static thermal averages. 


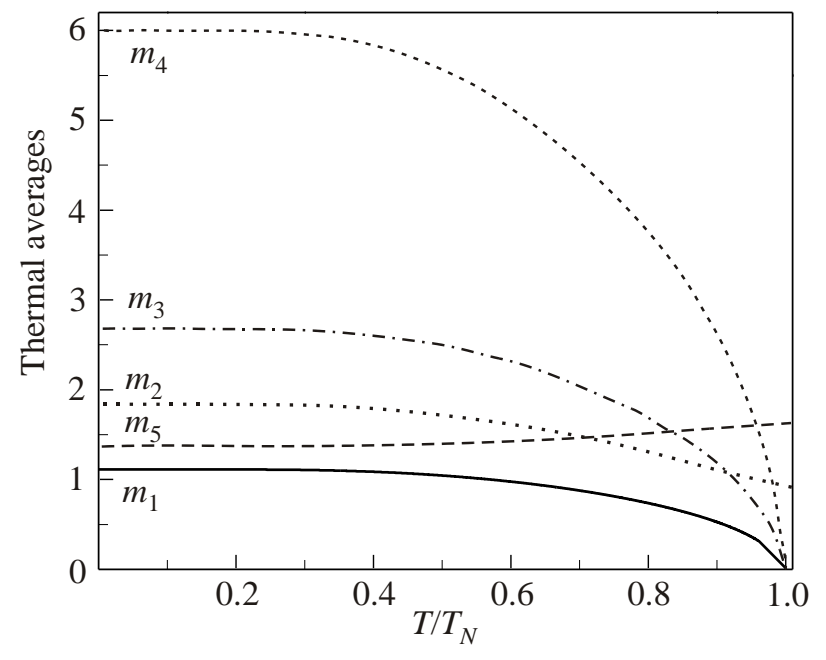

Fig. 9. (Color online) The various thermal averages defined in Eq. (12) versus reduced temperature $T / T_{N}$. The exchange and anisotropy parameters are the same as in Fig. 8.

\section{Comparison between experiment and theory}

Representative numerical results deduced from Eq. (10) for the low-temperature spin-wave energies versus wave vector are shown in Fig. 10, taking $\mathbf{k}=\left(0,0, k_{z}\right)$ with $k_{z}$ ranging across the Brillouin zone from 0 to $\pi / c$. The lowest-lying spin-wave excitation is generalized from the tran-

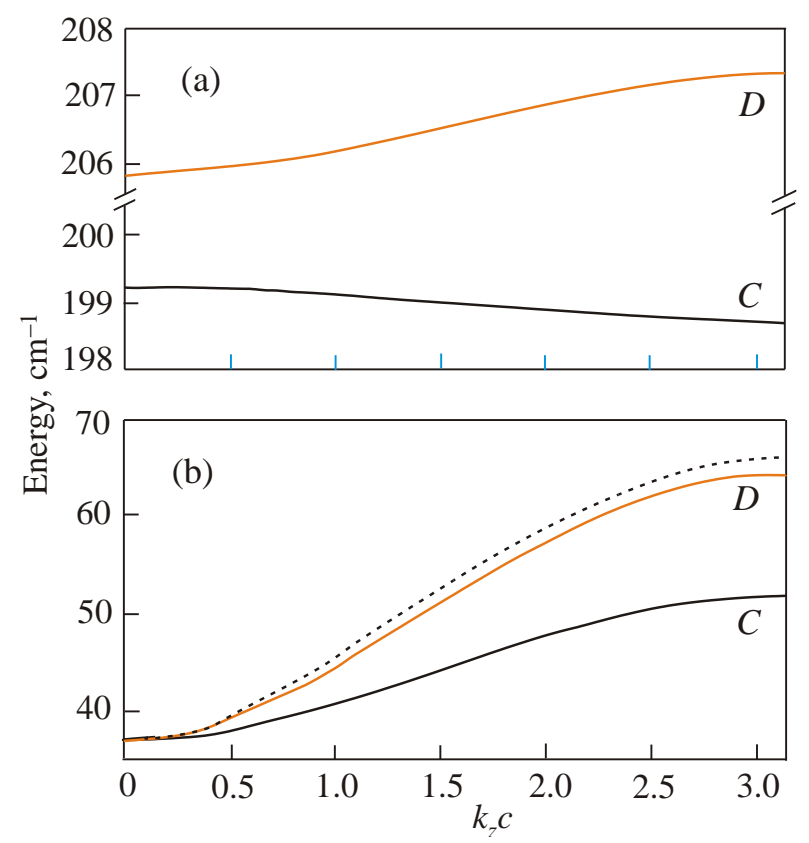

Fig. 10. (Color online) Spin-wave energy versus wave-vector component $k_{z}$. The excitation $E_{\delta}$ associated with the transition $\Delta_{\delta}$ (a) and low-lying spin-wave mode $E_{\alpha}$ associated with the transition $\Delta_{\alpha}$ (b) are shown. The solid lines are obtained using $J_{1}=0$ (labeled $C$ ) and $J_{1}=-2.0 \mathrm{~cm}^{-1}$ (labeled $D$ ). The dashed line corresponds to the results obtained using the effective spin $S=1 / 2$ model. sition $\Delta_{\alpha}$ from the mean-field ground state to the first excited level (see the lower panel), and another spin-wave branch associated with the transition $\Delta_{\delta}$ from the ground state to the third excited level (see the top panel) also exhibits dispersion. There are two additional spin-wave branches to the spectrum (which are effectively dispersionless and therefore not shown) that correspond to the transitions $\Delta_{\beta}$ and $\Delta_{\gamma_{1}}$ in Fig. 8; they correspond to energies of about $132.8 \mathrm{~cm}^{-1}$ and $14.2 \mathrm{~cm}^{-1}$, but are likely to be observed only at elevated temperatures (see later discussion) when there is sufficient thermal population of the higher levels in Fig. 8. In Fig. 10 we show the effect of varying the small intrasublattice exchange $J_{1}$ on the two excitations. We have also included in the lower panel, for comparison, the single spin-wave dispersion curve obtained using Eq. (4) in the effective spin $S=1 / 2$ model. The numerical results in this case are plotted using our optimal set of parameters for the $S=1 / 2$ model, i.e., $J_{1}=-1.2 \mathrm{~cm}^{-1}$, $J_{2}=12.9 \mathrm{~cm}^{-1}$, and $g \mu_{B} H_{A}(0)=12.0 \mathrm{~cm}^{-1}$.

For another comparison of the two theoretical approaches we show in Table 4 the spin-wave energies calculated using the $S=1 / 2$ and $S=3 / 2$ models at different points in the Brillouin zone. With these parameters the largest difference for the spin-wave energy $E_{\alpha}$ predicted by the two models occurs at the zone edge.

Table 4. Comparison of the low-temperature spin-wave energies (in $\mathrm{cm}^{-1}$ ) for different points in the Brillouin zone. Results for the spin $S=3 / 2$ model are obtained with $J_{1}=0 \mathrm{~cm}^{-1}$ in this example.

\begin{tabular}{c|c|c|c}
\hline Spin $S$ & Spin-wave branch & $\mathbf{k}=(0,0,0)$ & $\mathbf{k}=(0,0, \pi / c)$ \\
\hline \hline $3 / 2$ & $E_{\alpha}$ & 37.2 & 51.7 \\
& $E_{\beta}$ & 132.8 & 132.8 \\
& $E_{\gamma}$ & 199.2 & 198.7 \\
& $E_{\delta}$ & 14.2 & 14.2 \\
$1 / 2$ & $E_{\alpha}$ & 37.2 & 66.0 \\
\hline
\end{tabular}

However, the inelastic neutron scattering measurements [11] for the zone-edge excitation indicate a spin-wave excitation energy of $\approx 64.8 \mathrm{~cm}^{-1}$ which is comparable to the value obtained with the parameters of our effective spin $S=1 / 2$ model. With the small intrasublattice exchange $J_{1}=0$ the spin $S=3 / 2$ model underestimates the zone-edge spin-wave energy. The energy of the spin wave $E_{\alpha}$ at the zone edge may be increased with the inclusion of a nonzero $J_{1}$ term, as was done in Fig. 10. Thus, setting $J_{1}=-2.0 \mathrm{~cm}^{-1}$, the spinwave energy of $E_{\alpha}$ at $\mathbf{k}=(0,0, \pi / c)$ becomes $64.3 \mathrm{~cm}^{-1}$. The effect of the $J_{1}$ term on the dispersion of the excitation $E_{\gamma}$ is seen in Fig. 10(a), where $J_{1}$ produces a shift in the excitation energy as well as a change in sign of the slope of the curve.

In Fig. 11 we plot the $\mathbf{k}=0$ spin-wave energy $E_{\alpha}$ as a function of the reduced temperature for both the $S=1 / 2$ and $S=3 / 2$ models. The theoretical predictions are compared with new one-magnon Raman light scattering data. 


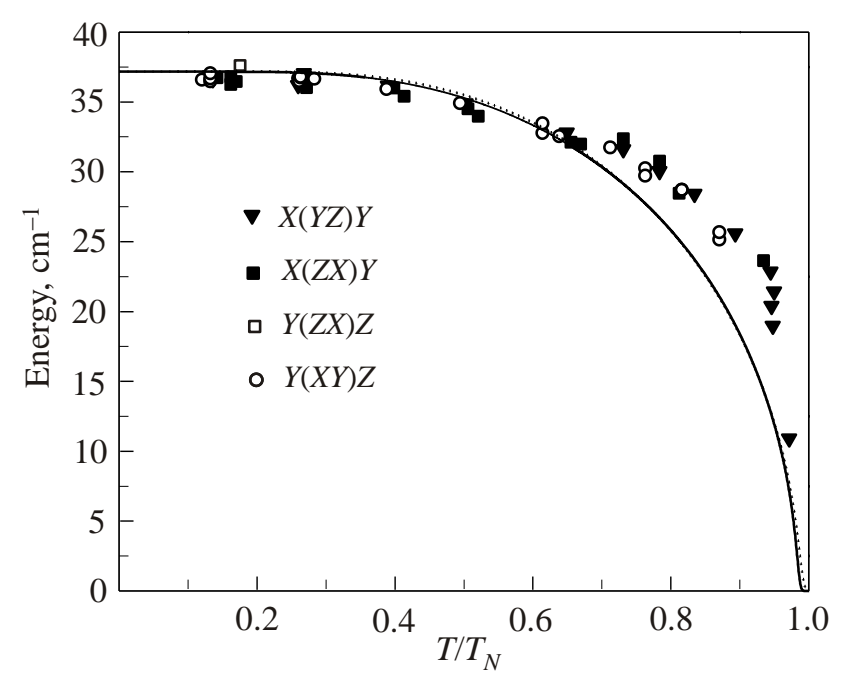

Fig. 11. Comparison of theory and experiment for the low-lying $\mathbf{k}=0$ excitations versus reduced temperature. The solid (dotted) line corresponds to results obtained using the spin $S=3 / 2(S=1 / 2)$ model.

Both models are in good agreement with experimental data for temperatures up to $T / T_{N}=0.6$. At higher temperatures the decoupling approximations used to linearize the equations of motion are no longer justified.

In Figs. 12(a) and (b) we show the excitation energies, calculated using the spin $S=3 / 2$ model, versus temperature for different fixed points in the Brillouin zone. As the temperature is increased the energies $E_{\alpha}$ and $E_{\gamma}$ eventually tend to zero because the splitting within the upper and lower doublets (see Fig. 8), which is produced by the exchange interactions, decreases with the sublattice magnetization. At the mean-field transition temperature the $E_{\beta}$ and $E_{\delta}$ excitations at $\mathbf{k}=0$ still have a small splitting but they become degenerate at the zone boundary as expected.

The formal results for the various spin-dependent Green's functions may straightforwardly be obtained by using $\mathbf{G}_{\mathbf{k}}=\left(E \mathbf{I}_{16}+\mathbf{B}\right)^{-1} \mathbf{b}$, which follows from Eq. (7). From standard relations between the spectral representation of the correlation functions and the Green's function we are able to extract information about the statistical weight associated with the various spin-wave excitations. As an illustrative example we consider the following transverse correlation function

$$
<S_{1 \mathbf{k}}^{+}(t) S_{1 \mathbf{k}}^{-}\left(t^{\prime}\right)>=\int_{-\infty}^{\infty} d E \exp \left[-i E\left(t-t^{\prime}\right)\right]<S_{1 \mathbf{k}}^{+} S_{1 \mathbf{k}}^{-}>_{E},
$$

here subscript 1 refers to the spin operators on the $i$-sublattice. The spectral function in Eq. (20) may then be written as

$$
\left.<S_{1 \mathbf{k}}^{+} S_{1 \mathbf{k}}^{-}\right\rangle_{E}=-2[n(E)+1] \operatorname{Im} \ll S_{1 \mathbf{k}}^{+} ; S_{1 \mathbf{k}}^{-} \gg_{E+i \varepsilon},
$$
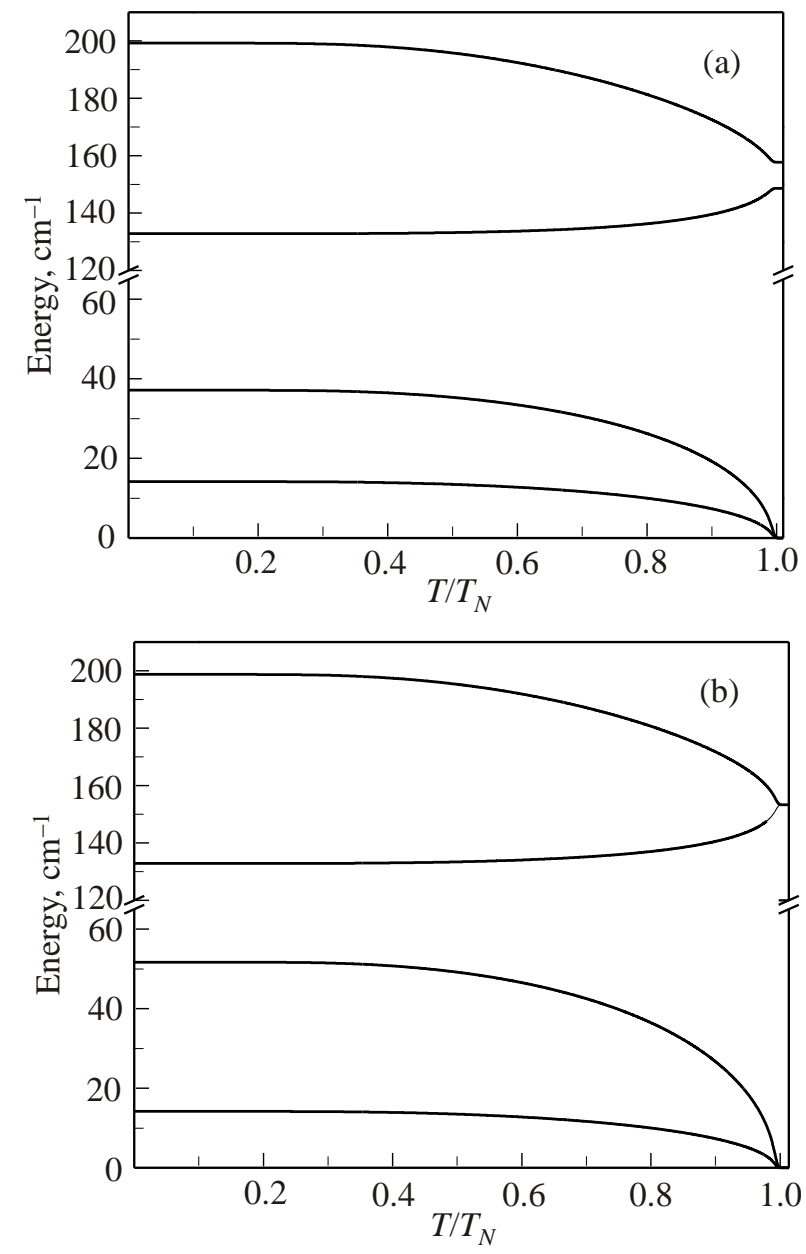

Fig. 12. Spin-wave energies versus $T / T_{N}$ for different values of the wavevector: zone-center $\mathbf{k}=(0,0,0)(\mathrm{a})$, zone-edge $\mathbf{k}=(0,0, \pi / \mathrm{c})(\mathrm{b})$.

where $n(E)$ is the Bose-Einstein thermal factor, $\varepsilon$ denotes a positive infinitesimal and the Green's function may be obtained from the solution of the inhomogenous matrix equation.

In Fig. 13 we show the spectral function, as defined in Eq. (21), for the various excitations predicted according to the $S=3 / 2$ model. In the low-temperature limit there is no statistical weight attached to the excitations $E_{\beta}$ and $E_{\gamma}$ since these modes involve transition between the higher energy states. However, at elevated temperatures (see the dashed lines) we predict a nonvanishing contribution from these excitations. The contribution to the spectral functions from the excitation $E_{\gamma}$ is found to be the smallest in this example. In the low-temperature limit the dominant spectral features come from the excitations $E_{\delta}$ (Fig. 13(b)) and $E_{\alpha}$ (Fig. 13(c)). These modes are associated with excitations from the ground state, which can occur even as $T \rightarrow 0$. At elevated temperatures the excitation peaks shift to lower values due to their dependence on the static thermal averages. 


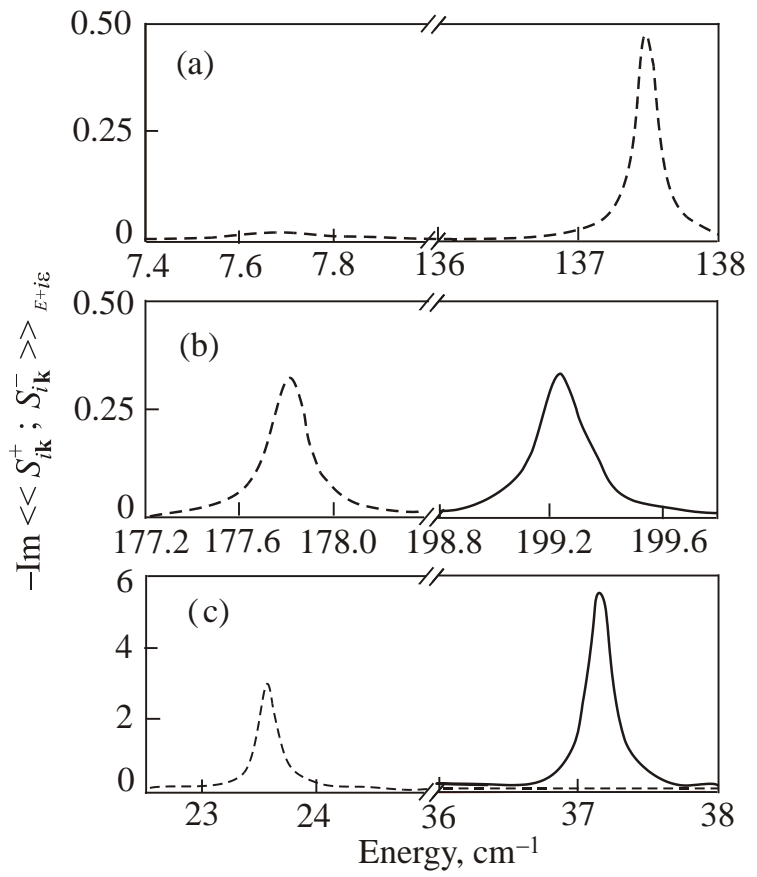

Fig. 13. Spectral function defined in Eq. (21) (excluding the thermal factor $2[n(E)+1])$ for the excitations $E_{\beta}$ and $E_{\gamma}(\mathrm{a}), E_{\delta}(\mathrm{b})$, and $E_{\alpha}(\mathrm{c})$. Here we have set $\mathbf{k}=0$ and $\varepsilon=0.1 \mathrm{~cm}^{-1}$. The solid and dashed lines correspond to results for $T \ll T_{N}$ and $T / T_{N}=0.6$, respectively.

\section{Excitations for $\Delta S^{z}= \pm 2$}

In Sec. 3 we investigated the magnetic excitations associated with the transitions $\Delta S^{z}= \pm 1$. They were just the one-magnon excitations deduced by studying spin operators that were linear in a transverse spin component. These operators were coupled through the equations of motion to other spin operators that were cubic in a transverse spin operator. However, the linearized equations of motion for these operators did not couple to any spin combinations that were quadratic (or any even power) in a transverse spin component. These latter operators are associated with the transitions having $\Delta S^{z}= \pm 2$. In this section we briefly investigate the properties of the excitations from the meanfield ground state to the second excited state, using the effective $S=3 / 2$ model. We note that the $S=1 / 2$ model is not capable of describing such excitations.

To study these modes we start from the equation of motion for $\ll\left(S_{i}^{+}\right)^{2} ; Y \gg_{E}$ and generate all of the other Green's functions coupled to it. As before we do not decouple the product of operators at the same site. Instead we form additional equations of motion in order to obtain a closed set. The equations of motion can be transformed to a wave vector representation and the set of equations may be written in a matrix form as $\left(E \mathbf{I}_{14}+B^{\prime}\right) \mathbf{G}_{\mathbf{k}}^{\prime}=\mathbf{b}^{\prime}$ where $\mathbf{I}_{14}$ is a $14 \times 14$ unit matrix, $\mathbf{G}_{\mathbf{k}}^{\prime}$ and $\mathbf{b}^{\prime}$ are 14-component column matrices whose elements are defined as in Eq. (7) with

$$
\begin{gathered}
X_{1}^{\prime}=\left(S_{i}^{+}\right)^{2}, \quad X_{2}^{\prime}=\left(S_{j}^{+}\right)^{2}, \quad X_{3}^{\prime}=\left(S_{i}^{-}\right)^{2}, \quad X_{4}^{\prime}=\left(S_{j}^{-}\right)^{2}, \\
X_{5}^{\prime}=\left(S_{i}^{+}\right)^{2} S_{i}^{z}+2 S_{i}^{+} S_{i}^{z} S_{i}^{+}+S_{i}^{z}\left(S_{i}^{+}\right)^{2}, \\
X_{6}^{\prime}=\left(S_{j}^{+}\right)^{2} S_{j}^{z}+2 S_{j}^{+} S_{j}^{z} S_{j}^{+}+S_{j}^{z}\left(S_{j}^{+}\right)^{2}, \\
X_{7}^{\prime}=\left(S_{i}^{-}\right)^{2} S_{i}^{z}+2 S_{i}^{-} S_{i}^{z} S_{i}^{-}+S_{i}^{z}\left(S_{i}^{-}\right)^{2}, \\
X_{8}^{\prime}=\left(S_{j}^{-}\right)^{2} S_{j}^{z}+2 S_{j}^{-} S_{j}^{z} S_{j}^{-}+S_{j}^{z}\left(S_{j}^{-}\right)^{2}, \\
X_{9}^{\prime}=\left(S_{i}^{z}\right)^{3}, \quad X_{10}^{\prime}=\left(S_{j}^{z}\right)^{3}, \quad X_{11}^{\prime}=3\left(S_{i}^{z}\right)^{2}-15 / 4, \\
X_{12}^{\prime}=3\left(S_{j}^{z}\right)^{2}-15 / 4, \quad X_{13}^{\prime}=4\left(S_{i}^{z}\right)^{3}-13 S_{i}^{z}, \\
X_{14}^{\prime}=4\left(S_{j}^{z}\right)^{3}-13 S_{j}^{z} .
\end{gathered}
$$

The nonzero elements of the $14 \times 14$ matrix $\mathbf{B}^{\prime}$ are defined in the Appendix.

Here, for simplicity, we did not include the effects of the intrasublattice exchange interaction $J_{1}$. Note that the equations of motion also involve some of the static thermal averages which were defined in Eq. (12). The subset $\left(X_{1}^{\prime}, \ldots, X_{8}^{\prime}\right)$ of the operators defined above in Eq. (22) are quadratic in the transverse spin component. They give rise to magnetic excitations (denoted by $E_{\sigma}$ and $E_{\tau}$ ) that are associated with the transitions labelled as $\Delta_{\sigma}$ and $\Delta_{\tau}$ in Fig. 14. The equations of motion are also coupled to combinations of operators involving products of the longitudinal spin component $S^{z}$. The equations of motion for these operators do not vanish because of the nonuniaxial anisotropy term in the Hamiltonian.

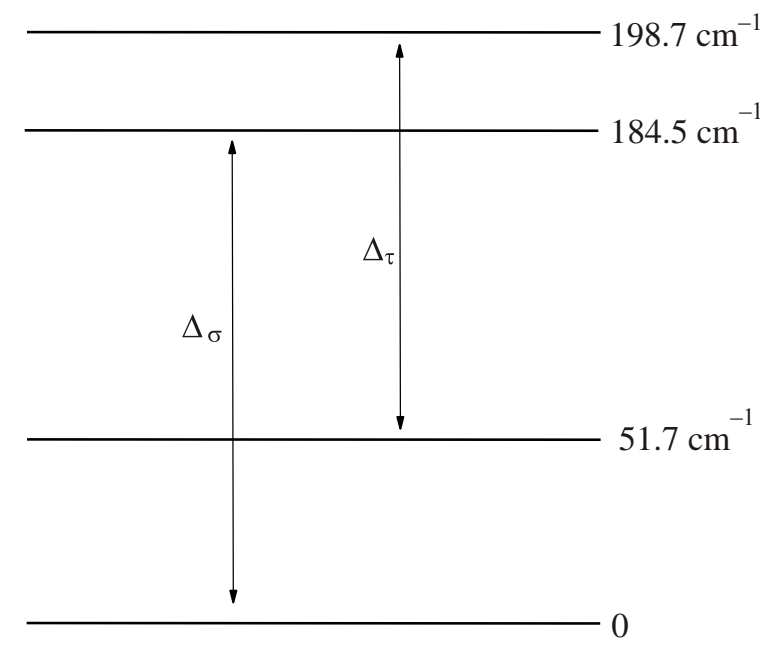

Fig. 14. Schematic representation of the energy levels of the $\mathrm{Co}^{2+}$ magnetic ions, following Fig. 8. The transitions now labelled as $\Delta_{\sigma}$ and $\Delta_{\tau}$ correspond to the selection rules $\Delta S^{z}= \pm 2$. 
In Fig. 15 we show the magnetic excitations obtained from the determinantal condition $\operatorname{det}\left(E \mathbf{I}_{14}+\mathbf{B}^{\prime}\right)=0$ plotted versus wave vector $k_{z}$. The magnetic excitation $E_{\sigma}$ (upper two curves) is split at the zone center due to the exchange interaction but becomes degenerate at the zone edge. The splitting of this mode has been observed experimentally [11] and the measured excitations energies at $\mathbf{k}=0$ are 170 and $206 \mathrm{~cm}^{-1}$, compared with 165 and $203 \mathrm{~cm}^{-1}$ respectively from the theory. At $\mathbf{k}=(0,0, \pi / c)$ the observed excitation energy is $190.1 \mathrm{~cm}^{-1}$, compared with $185 \mathrm{~cm}^{-1}$ from the theory. The dispersionless branch at $147 \mathrm{~cm}^{-1}$ corresponds to the excitation $E_{\tau}$ in Fig. 10. In the low-temperature region the statistical weight attached to this mode is small because it involves excitation between higher energy states.

\section{Conclusions}

In this paper we have investigated the magnetic excitations in a spin $S=3 / 2$ anisotropic antiferromagnet with strong spin-orbit coupling. Detailed experimental results are presented for the temperature and polarization dependence of the one-magnon Raman scattering in the rutile structure antiferromagnet $\mathrm{CoF}_{2}$. Low temperature results are also presented and discussed for Raman scattering from higher-energy excitons in the ground term. The Green's function equation of motion method was employed to derive the excitation energies and spectral intensities over a broad range of temperatures. Results were obtained using RPA for the product of operators at different sites while the single-ion anisotropy terms were treated exactly (without using any decoupling scheme) by generating a closed set of coupled Green function equations. The theory was applied to $\mathrm{CoF}_{2}$ and the numerical results were compared with one-magnon Raman light scattering data reported

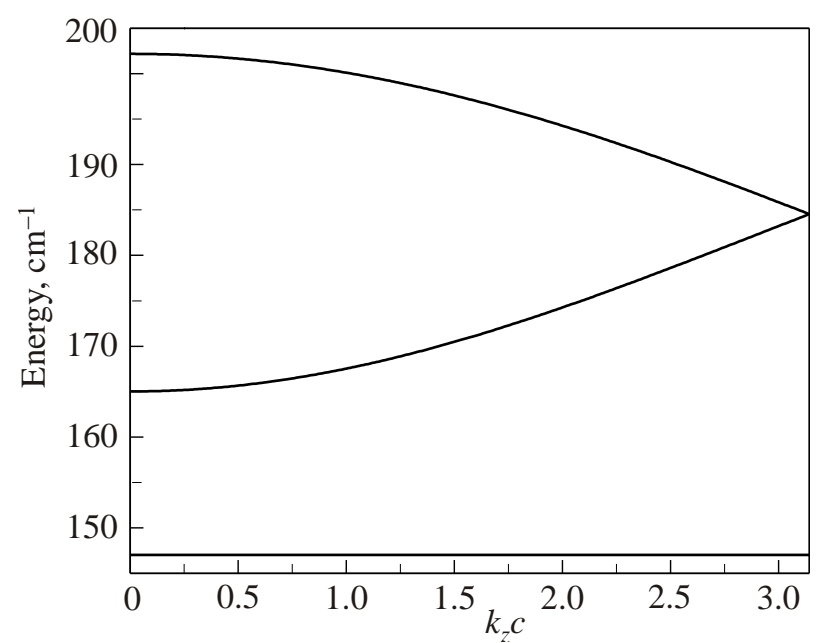

Fig. 15. Magnetic excitations $E_{\sigma}$ (upper two branches) and $E_{\tau}$ (lowest flat branch) involving the selection rules $\Delta S^{z}= \pm 2$ versus wave vector $k_{z} c$. The excitation energies are calculated using the same parameter values as in Fig. 8 .

here, as well as other published works. At elevated temperatures the theory predicts several optical magnetic excitations associated with transitions between the higher energy magnetic states. The statistical weight attached to these optical modes vanishes in the $T=0$ limit. The dispersion and temperature dependences of the lowest-lying excitation using the spin $S=3 / 2$ model were also compared to results obtained using a simpler effective spin $S=1 / 2$ model.

This work was partially supported by the Natural Sciences and Engineering Research Council (NSERC) of Canada. We thank P.A. Moch for helpful discussions, J. Johnson for assistance in the analysis of the Raman spectra, and H.J. Labbé for the crystal sample preparation.

\section{Appendix: Matrix elements in the $S=3 / 2$ model}

The nonzero elements of the $16 \times 16$ matrix $\mathbf{B}$ appearing in Eq. (7) are given by

$$
\begin{gathered}
B_{1,1}=-B_{2,2}=-B_{3,3}=B_{4,4}=-J_{2}(0) m_{1}+\left(J_{1}(0)+J_{3}(0)-J_{1}(\mathbf{k})-J_{3}(\mathbf{k})\right) m_{1}, \\
B_{1,2}=-B_{2,1}=-B_{3,4}=B_{4,3}=-J_{2}(\mathbf{k}) m_{1}, \\
B_{1,5}=B_{2,6}=-B_{3,7}=-B_{4,8}=B_{5,9}=B_{6,10}=-B_{7,11}=-B_{8,12}=\frac{1}{4} B_{9,5}=\frac{1}{4} B_{10,6}=-\frac{1}{4} B_{11,7}=-\frac{1}{4} B_{12,8}=D, \\
B_{1,7}=-B_{2,8}=-B_{3,5}=B_{4,6}=\frac{2}{3} B_{5,11}=-\frac{2}{3} B_{6,12}=-\frac{2}{3} B_{7,9}=\frac{2}{3} B_{8,10}=\frac{1}{2} B_{5,13}=-\frac{1}{2} B_{6,14}= \\
=-\frac{1}{2} B_{7,15}=\frac{1}{2} B_{8,16}=\frac{1}{3} B_{13,5}=-\frac{1}{3} B_{14,6}=-\frac{1}{3} B_{15,7}=\frac{1}{3} B_{16,8}=-F, \\
B_{5,1}=B_{6,2}=-B_{7,3}=-B_{8,4}=-\left(J_{1}(\mathbf{k})+J_{3}(\mathbf{k})\right) m_{2}, \\
B_{5,2}=B_{6,1}=-B_{7,4}=-B_{8,3}=-J_{2}(\mathbf{k}) m_{2}, \\
B_{5,3}=-B_{6,4}=-B_{7,1}=B_{8,2}=6 F-\left(J_{1}(\mathbf{k})+J_{3}(\mathbf{k})\right) m_{5},
\end{gathered}
$$




$$
\begin{gathered}
B_{5,4}=-B_{6,3}=-B_{7,2}=B_{8,1}=-J_{2}(\mathbf{k}) m_{5}, \\
B_{5,5}=-B_{6,6}=-B_{7,7}=B_{8,8}=B_{9,9}=-B_{10,10}=-B_{11,11}=B_{12,12}= \\
=\frac{1}{3} B_{13,13}=-\frac{1}{3} B_{14,14}=-\frac{1}{3} B_{15,15}=\frac{1}{3} B_{16,16}=-J_{2}(0) m_{1}+\left(J_{1}(0)+J_{3}(0)\right) m_{1}, \\
B_{9,1}=-B_{10,2}=-B_{11,3}=B_{12,4}=-\left(J_{1}(\mathbf{k})+J_{3}(\mathbf{k})\right) m_{4}, \\
B_{9,2}=-B_{10,1}=-B_{11,4}=B_{12,3}=-J_{2}(\mathbf{k}) m_{4}, \\
B_{9,3}=B_{10,4}=-B_{11,1}=-B_{12,2}=\frac{4}{3} B_{13,2}=\frac{4}{3} B_{14,1}=-\frac{4}{3} B_{15,4}=-\frac{4}{3} B_{16,3}=-\left(J_{1}(\mathbf{k})+J_{3}(\mathbf{k})\right) m_{3}, \\
B_{9,4}=B_{10,3}=-B_{11,2}=-B_{12,1}=\frac{4}{3} B_{13,1}=\frac{4}{3} B_{14,2}=-\frac{4}{3} B_{15,3}=-\frac{4}{3} B_{16,4}=-J_{2}(\mathbf{k}) m_{3} .
\end{gathered}
$$

The nonzero elements of the $14 \times 14$ matrix $\mathbf{B}^{\prime}$ arising in the discussion of the $\Delta S^{z}= \pm 2$ magnetic excitations are

$$
\begin{gathered}
B_{1,1}^{\prime}=-B_{2,2}^{\prime}=-B_{3,3}^{\prime}=B_{4,4}^{\prime}=B_{5,5}^{\prime}=-B_{6,6}^{\prime}=-B_{7,7}^{\prime}=B_{8,8}^{\prime}=-2 J_{2}(0) m_{1}, \\
B_{1,5}^{\prime}=B_{2,6}^{\prime}=-B_{3,7}^{\prime}=-B_{4,8}^{\prime}=\frac{1}{4} B_{5,1}^{\prime}=\frac{1}{4} B_{6,2}^{\prime}=-\frac{1}{4} B_{7,3}^{\prime}=-\frac{1}{4} B_{8,4}^{\prime}=D, \\
B_{1,10}^{\prime}=-B_{2,9}^{\prime}=-B_{3,10}^{\prime}=B_{4,9}^{\prime}=2 J_{2}(\mathbf{k}) m_{5}, \\
B_{1,13}^{\prime}=-B_{2,14}^{\prime}=-B_{3,13}^{\prime}=B_{4,14}^{\prime}=-\frac{1}{4} B_{5,11}^{\prime}=\frac{1}{4} B_{6,12}^{\prime}=\frac{1}{4} B_{7,11}^{\prime}=-\frac{1}{4} B_{8,12}^{\prime}=-B_{9,1}^{\prime}=B_{9,3}^{\prime}=B_{10,2}^{\prime}=-B_{10,4}^{\prime}= \\
=-\frac{2}{3} B_{11,5}^{\prime}=\frac{2}{3} B_{11,7}^{\prime}=\frac{2}{3} B_{12,6}^{\prime}=-\frac{2}{3} B_{12,8}^{\prime}=\frac{1}{6} B_{13,1}^{\prime}=-\frac{1}{6} B_{13,3}^{\prime}=-\frac{1}{6} B_{14,2}^{\prime}=\frac{1}{6} B_{14,4}^{\prime}=F, \\
B_{5,10}^{\prime}=B_{6,9}^{\prime}=-B_{7,10}^{\prime}=-B_{8,9}^{\prime}=2 J_{2}(\mathbf{k}) m_{3} .
\end{gathered}
$$

1. E. Meloche, M.G. Cottam, and D.J. Lockwood, Phys. Rev. B 76, 104406 (2007).

2. M.G. Cottam and D.J. Lockwood, Light Scattering in Magnetic Solids, Wiley, New York (1986).

3. E. Meloche, M.G. Cottam, V.P. Gnezdilov, and D. Lockwood, Phys. Rev. B 81, 024426 (2010).

4. R.A. Erickson, Phys. Rev. 90, 779 (1953).

5. J.W. Stout and L.M. Matarrese, Rev. Mod. Phys. 25, 338 (1953).

6. T. Nakamura and H. Taketa, Prog. Theor. Phys. 13, 129 (1955).

7. M.E. Lines, Phys. Rev. 137, A982 (1965).

8. P.L. Richards, J. Appl. Phys. 35, 850 (1964).

9. R. Newman and R.M. Chrenko, Phys. Rev. 115, 1147 (1959).

10. H.M. Gladney, Phys. Rev. 146, 253 (1966).

11. R.A. Cowley, P. Martel, and R.W.H. Stevenson, Phys. Rev. Lett. 18, 162 (1967).
12. P. Martel, R.A. Cowley, and R.W.H. Stevenson, Can. J. Phys. 46, 1355 (1968).

13. R.M. Macfarlane, Phys. Rev. Lett. 25, 1454 (1970).

14. A.P. Moch, J.-P. Gosso, and C. Dugautier, in: Light Scattering in Solids, M. Balkanski (ed.), Flammarion, Paris (1971), p. 138.

15. B.J.T. Hoff, P.A. Grünberg, and J.A. Koningstein, Appl. Phys. Lett. 20, 358 (1972).

16. A. Ishikawa and T. Moriya, J. Phys. Soc. Jpn. 30, 117 (1971).

17. S.J. Allen, Jr., and H.J. Guggenheim, J. Appl. Phys. 42, 1657 (1971).

18. S.J. Allen, Jr., and H.J. Guggenheim, Phys. Rev. B 4, 937 and 950 (1971).

19. L.J. Zimring and J.W. Stout, J. Chem. Phys. 51, 4197 (1969).

20. D.J. Lockwood and Z.R. Wasilewski, Phys. Rev. B 70, 155202 (2004).

21. M.G. Cottam and A. Latiff Awang, J. Phys. C 10, 3673 (1977). 